\title{
Different aggregation and shape characteristics of carbon materials affect biological responses in RAW264 cells
}

This article was published in the following Dove Press journal: International Journal of Nanomedicine

\begin{abstract}
Chika Kuroda, ${ }^{1,2, *}$ Katsuya Ueda, ${ }^{1,3, *}$ Hisao Haniu, ${ }^{1,3-5}$ Haruka Ishida, ${ }^{1,4}$ Satomi Okano, ${ }^{1,4}$ Takashi Takizawa, ${ }^{5}$ Atsushi Sobajima, ${ }^{5}$ Takayuki Kamanaka, ${ }^{5}$ Kazushige Yoshida, ${ }^{5}$ Masanori Okamoto, ${ }^{5}$ Tamotsu Tsukahara, ${ }^{6}$ Yoshikazu Matsuda, ${ }^{7}$ Kaoru Aoki, ${ }^{8}$ Hiroyuki Kato, ${ }^{5}$ Naoto Saito ${ }^{1,3-5}$

'Institute for Biomedical Sciences, Interdisciplinary Cluster for Cutting Edge Research, Shinshu University, Asahi, Matsumoto, Nagano, Japan; ${ }^{2}$ Department of Orthopaedic Surgery, Graduate School of Medicine, Shinshu University, Asahi, Matsumoto, Nagano, Japan; ${ }^{3}$ Department of Biomedical Engineering, Graduate School of Science and Technology, Shinshu University, Asahi, Matsumoto, Nagano, Japan; ${ }^{4}$ Department of Biomedical Engineering, Graduate School of Medicine, Science and Technology, Shinshu University, Asahi, Matsumoto, Nagano, Japan; ${ }^{5}$ Department of Orthopaedic Surgery, Shinshu University School of Medicine, Asahi, Matsumoto, Nagano, Japan; 'Department of Molecular Pharmacology and Neuroscience, Nagasaki University Graduate School of Biomedical Sciences, Bunkyo-machi, Nagasaki, Japan ${ }^{7}$ Clinical Pharmacology Educational Center, Nihon Pharmaceutical University, Komuro, Ina-machi, Saitama, Japan; ${ }^{8}$ Physical Therapy Division, School of Health Sciences, Shinshu University, Asahi, Matsumoto, Nagano, Japan

*These authors contributed equally to this work
\end{abstract}

Correspondence: Hisao Haniu Institute for Biomedical Sciences, Interdisciplinary Cluster for Cutting Edge Research, Shinshu University, 3-I-I Asahi, Matsumoto, Nagano, Japan

Tel +8I 263373555

Fax +81263373549

Email hhaniu@shinshu-u.ac.jp
Introduction: Carbon nanotubes (CNTs) have various shapes, including needle-like shapes and curled shapes, and the cytotoxicity and carcinogenicity of CNTs differ depending on their shapes and surface modifications. However, the biological responses induced by CNTs and related mechanisms according to the dispersion state of CNTs have not been extensively studied.

Materials and methods: We prepared multiwalled CNTs (MWCNTs) showing different dispersions and evaluated these MWCNTs in RAW264 cells to determine cytotoxicity, cellular uptake, and immune responses. Furthermore, RAW264 cells were also used to compare the cellular uptake and cytotoxicity of fibrous MWCNTs and spherical carbon nanohorns (CNHs) exhibiting the same degree of dispersion.

Results: Our analysis showed that the cellular uptake, localization, and inflammatory responses of MWCNTs differed depending on the dispersion state. Moreover, there were differences in uptake between MWCNTs and CNHs, even showing the same degree of dispersion. These findings suggested that receptors related to cytotoxicity and immune responses differed depending on the aggregated state of MWCNTs and surface modification with a dispersant. Furthermore, our results suggested that the receptors recognized by the cells differed depending on the particle shape.

Conclusion: Therefore, to apply MWCNTs as a biomaterial, it is important to determine the carcinogenicity and toxicity of the CNTs and to examine different biological responses induced by varying shapes, dispersion states, and surface modifications of particles.

Keywords: multiwalled carbon nanotubes, aggregation, carbon nanohorns, cytotoxicity, immune response, cellular uptake

\section{Introduction}

Carbon nanotubes (CNTs) are nanomaterials having a structure in which graphene sheets are wound in a tubular shape. CNTs have unique electrical characteristics, thermal properties, and mechanical properties ${ }^{1,2}$ because of their composition (only carbon atoms) and specific shape, and by combining CNTs with other materials, the performance of the base metal is greatly improved. Thus, product development in various fields has been actively carried out, ${ }^{3}$ such as in radionuclide therapy ${ }^{4}$ and bioimaging studies, ${ }^{5}$ and we have developed sockets for artificial joints using tangled multiwalled CNTs (MWCNTs). However, the safety of MWCNTs when used for applications in human beings is still unclear, and further studies are needed to confirm the safety of MWCNTs for use in living organisms. ${ }^{6}$

Because MWCNTs are foreign substances in the living body, the immune system is activated acutely in their presence, and MWCNTs are then phagocytosed by immune cells, such as macrophages. ${ }^{7}$ Uptake of foreign substances by these cells 
represents a toxic stimulus, resulting in an inflammatory reaction and subsequent tissue fibrosis. ${ }^{8,9}$ MWCNTs incorporated into macrophages induce the expression of immune system proteins, stimulating the secretion of inflammatory cytokines. ${ }^{10,11}$ In contrast, some studies have shown that exposure of MWCNTs to macrophages does not induce a strong inflammatory response. ${ }^{12-14}$ Further studies have shown that the immune response to cells following the exposure to MWCNTs varies according to both surface modifications and MWCNT size. ${ }^{15}$ In addition, MWNT7, a type of MWCNTs, has a structure resembling a needle, similar to asbestos, and shows obvious carcinogenicity. ${ }^{16,17}$ In contrast, tangled MWCNTs, which are different from the needle-like MWNT7, have been reported to exhibit no carcinogenicity in rats. Therefore, not all CNTs show carcinogenic properties, but CNT size and shape may be related to carcinogenicity.

Untreated CNTs are hydrophobic and have low solubility; therefore, they tend to aggregate in aqueous solution. Furthermore, tangled CNTs, which have not yet been shown to be carcinogenic, can become entangled due to their diameter (several nanometers to several tens of nanometers) and length (up to several micrometers). In previous studies, nondispersive CNTs have been shown to form larger aggregates and show stronger toxicity. ${ }^{15}$ However, some studies have successfully dispersed CNTs completely and evaluated the CNT particles themselves. ${ }^{18,19}$ In general, strong ultrasonic treatment is necessary to achieve sufficient dispersion, but can cause shearing of the CNTs. ${ }^{20}$

In previous studies from our laboratory, highly dispersed CNTs were fabricated using ultrasonic treatment equipment that did not change the length of CNTs by applying medium ultrasonic waves while rotating the sample. Evaluation of highly dispersed CNTs and aggregated tangled CNTs using carcinogenic human bronchus cells demonstrated that only aggregated CNTs were taken up by cells and showed growth suppression. ${ }^{21}$ However, for macrophages, which are believed to affect the carcinogenicity of CNTs, ${ }^{19}$ few studies have investigated the effects of fully dispersed tangled CNTs on cell proliferation and immune responses.

In this study, we aimed to clarify the immune responses of highly dispersed CNTs and aggregated CNTs using RAW264 cells as mouse macrophage-like cells. Furthermore, we compared and investigated the influence of the dispersion state, fibrous CNTs, and spherical carbon nanohorns (CNHs) on the immune response.

\section{Materials and methods} Characterization of MWCNTs and CNHs MWCNT materials were provided by Cnano Technology (FT9110; Santa Clara, CA, USA). FT9110 was manufactured using a catalytic vapor deposition method and had specific properties as described by the manufacturer (diameter: $10-15 \mathrm{~nm}$, length: $10 \mu \mathrm{m}$ or less, purity: $99.8 \%$ or more, iron content: $<100 \mathrm{ppm})$.

CNHs (CNHox; NEC, Tokyo, Japan) had specific properties as described by the manufacturer (diameter of aggregates: approximately $100 \mathrm{~nm}$, purity: $95 \%$ or more, graphene content: $<5 \%$, iron content: $0 \%$ ).

\section{Suspension and dispersion of FT9IIO and $\mathrm{CNHs}$}

FT9110 was sterilized in an autoclave at $121^{\circ} \mathrm{C}$ for 15 minutes and dried, and $10 \mathrm{mg} / \mathrm{mL}$ FT9110 was then vortexed in two dispersants (2\% FBS [Biowest, Nuaillé, France] in Dulbecco's PBS [DPBS] and 0.1\% PS [NOF, Tokyo, Japan] in DPBS). FT9110 was dispersed using three different sonicators that were renamed according to the ultrasonic treatment method and output: the PR-1 sonicator (Thinky, Tokyo, Japan) was termed as W-140 (water bath type, $140 \mathrm{~W}$ power), the US-1R sonicator (As one, Tokyo, Japan) was termed as W-55 (water bath type, $55 \mathrm{~W}$ power), and the W-220 sonicator (Heat Systems-Ultrasonic, Plainview, NY, USA) was termed as $\mathrm{H}-140$ (homogenizer type, $140 \mathrm{~W}$ power). $\mathrm{CNHs}$ were dispersed at $1 \mathrm{mg} / \mathrm{mL}$ by sonication using W-140.

To determine the hydrodynamic size of the agglomerated FT9110 and CNHs, sonicated FT9110 and CNHs were measured using a Zetasizer Nano ZS (Malvern Instruments, Malvern, UK; Table 1), and the zeta potential of the sonicated FT9110 and CNHs was determined with a Zetasizer Nano ZS (Table 2). The FT9110 and CNHs were diluted to $0.1 \mathrm{mg} / \mathrm{mL}$, and each measurement was conducted in triplicate.

\section{Cell culture}

RAW264 mouse monocyte macrophages were purchased from Riken (Saitama, Japan). RAW264 cells were cultured in minimum essential medium (MEM; Nacalai Tesque, Kyoto, Japan) with $10 \%$ FBS and nonessential amino acid solution (100×; Nacalai Tesque) at $37^{\circ} \mathrm{C}$ in a $5 \% \mathrm{CO}_{2}$ humidified incubator and passaged twice per week. For each experiment, the

Table I Diameter of FT9IIO and CNH particles used in the experiments

\begin{tabular}{|c|c|c|c|}
\hline \multirow[t]{2}{*}{ Material } & \multirow[t]{2}{*}{ Sonicator } & \multicolumn{2}{|l|}{ Dispersant } \\
\hline & & FBS (nm) & PS (nm) \\
\hline \multirow[t]{3}{*}{ CNTs } & $W-140$ & $188 \pm 72$ & $135 \pm 49$ \\
\hline & W-55 & $4,434 \pm 34 \mid$ & $5,100 \pm 488$ \\
\hline & $\mathrm{H}-\mathrm{I} 40$ & $1,603 \pm 113$ & $2,78 I \pm 5 \mid 2$ \\
\hline $\mathrm{CNHs}$ & $W-140$ & $|72 \pm 6|$ & $\mid 46 \pm 48$ \\
\hline
\end{tabular}

Abbreviations: $\mathrm{CNH}$, carbon nanohorn; CNT, carbon nanotube; FT9I 10, Flotube 9110; PS, polysorbate 80. 
Table 2 Zeta potential of FT9I 10 and $\mathrm{CNH}$ particles used in the experiments

\begin{tabular}{llll}
\hline Material & Sonicator & \multicolumn{2}{l}{ Dispersant } \\
\cline { 3 - 4 } & & FBS $(\mathbf{m V})$ & PS $(\mathbf{m V})$ \\
\hline CNTs & W- 140 & $-14.6 \pm 0.3$ & $-4.3 \pm 0.2$ \\
& W-55 & $-12.9 \pm 0.5$ & $-4.3 \pm 0.2$ \\
& $\mathrm{H}-140$ & $-13.4 \pm 0.4$ & $-6.3 \pm 0.3$ \\
$\mathrm{CNHs}$ & $\mathrm{W}-140$ & $-13.4 \pm 0.6$ & $-8.7 \pm 0.4$ \\
\hline
\end{tabular}

Abbreviations: $\mathrm{CNH}$, carbon nanohorn; CNTs, carbon nanotubes; FT9II0, Flotube 91 10; PS, polysorbate 80.

cells were seeded at a density of $6 \times 10^{4}$ cells $/ \mathrm{mL}$ and allowed to adhere for 24 hours.

\section{Cell viability}

Cell viability was assessed using an alamar blue assay (alamarBlue ${ }^{\circledR}$ cell viability reagent; Remel, Lenexa, KS, USA). Cells were plated in 96-well plates and incubated for 24 or 48 hours at $37^{\circ} \mathrm{C}$ in culture medium containing $100 \mu \mathrm{g} / \mathrm{mL}$ FT9110 in a dispersant or in control medium containing only dispersant. After aspiration of the culture medium to exclude the influence of FT9110, 10\% alamar blue reagent in culture medium was added to each well, where viable cells metabolized the dye for 60 minutes, resulting in increased fluorescence detected by excitation/emission at 535/590 nm using a plate reader (AF2200; Eppendorf, Hamburg, Germany). Cell viability was calculated as follows: percent cytotoxicity $=$ $100 \times$ experimental value/control value. The media were assayed six times for each treatment condition.

\section{Observation of cells by fluorescence microscopy}

Cells cultured on Cellview glass-bottomed advanced TC four compartments (Greiner Bio-one, Frickenhausen, Germany) were exposed to FT9110 and CNHs at $100 \mu \mathrm{g} / \mathrm{mL}$ for 48 hours under the same conditions as described for the cell viability assay. For assessment, cells were stained with nuclear stain (Bisbenzimide H33342 Fluorochrome Trihydrochloride Dimethyl Sulfoxide Solution; Nacalai Tesque) and lysosome stain (Cyto Painter Lysosomal Staining ab 138895; Abcam, Cambridge, UK) 30 minutes before observation. The cells were visualized using an AxioObserverZ1 fluorescence microscope (Carl Zeiss Jena GmbH, Jena, Germany) with a $40 \times$ objective lens.

\section{Assessment of FT9I I 0 uptake by flow cytometry}

Assessment of FT9110 uptake was determined by flow cytometry. Briefly, the cells were grown in 24-well plates incubated for 24 hours at $37^{\circ} \mathrm{C}$ in the presence or absence of $10 \mu \mathrm{g} / \mathrm{mL}$ FT9110. The cells were washed with DPBS to remove unbound FT9110, harvested with trypsin, and centrifuged at $400 \times g$ for 3 minutes. The precipitated cells were suspended in DPBS containing FBS. Side scatter in a light-scattering analysis was immediately measured up to 10,000 events using a FACSCalibur instrument. Test media were assayed in triplicate for each treatment condition.

\section{Observation by electron microscopy}

Cells grown on cover slips in six-well plates were exposed to FT9110 and CNHs (100 $\mu \mathrm{g} / \mathrm{mL}$ in RAW264 cells) for 24 hours. Cells were washed twice in DPBS, fixed with $2.5 \%$ glutaraldehyde, postfixed with $1 \%$ osmic acid, and embedded in Epon embedding resin (Epok 812; Okenshoji, Tokyo, Japan). Sections were cut to $60 \mathrm{~nm}$ thickness, stained with uranyl acetate and lead citrate, and visualized under a JEM1400 TEM (JEOL, Tokyo, Japan) at $80 \mathrm{keV}$.

\section{Cytokine measurement}

Cytokines in the culture supernatant were measured with a cytometric bead array flex set system (BD Biosciences, San Jose, CA, USA), according to the manufacturer's protocol. Briefly, RAW264 cells in 24-well plates were exposed to $10 \mu \mathrm{g} / \mathrm{mL}$ FT9110 for 24 hours, and cytokine capture beads (for TNF, RANTES, MIP-1 $\alpha$, MCP-1, IL-1 $\beta$, IL-10, and IL-6) were added to the samples or cytokine standards $(10-2,500 \mathrm{pg} / \mathrm{mL})$ in flow cytometry tubes. The mixtures were vortexed, and antibodies for fluorescence detection were added to each tube. The samples were then incubated at room temperature for 2 hours. Following incubation, the beads were washed once and resuspended prior to reading with a FACSCalibur apparatus (BD Biosciences). Test media were assayed in triplicate for each treatment condition.

\section{Statistical analysis}

Data are presented as mean \pm standard error (SE). Statistical significance was determined by analysis of variance followed by the Tukey-Kramer method. Differences with $P$-values of $<0.05$ were considered statistically significant.

\section{Results Cell viability}

The viability of cells exposed to Flotube 9110 (FT9110) dispersed in polysorbate 80 (PS) for 24 hours was significantly decreased compared with that of the control, and significant differences were observed between the effects of particles dispersed using the W-140 and the W-55 or H-140 
sonicators (W-140: 93.9\%, W-55: 64.1\%, H-140: 65.4\%). After 48 hours, the cell survival rate further decreased with W-55 and H-140 (W-140: 98.9\%, W-55: 48.2\%, H-140: 42.2\%). Cells exposed to FT9110 dispersed with FBS showed $104.4 \%, 78.6 \%$, and $94.1 \%$ viability using W-140, W-55, and H-140, respectively, at 24 hours and $126.5 \%, 82.3 \%$, and $76.4 \%$ viability using $\mathrm{W}-140, \mathrm{~W}-55$, and $\mathrm{H}-140$, respectively, at 48 hours; thus, there were no decreases in cell viability when compared with the control group (Figure 1).

\section{Observation of cells by fluorescence microscopy}

Next, we examined the state of cells exposed to FT9110 with a fluorescent microscope. The cells exposed to FT9110 dispersed using the W-140, regardless of the dispersant, were adhered to the glass bottom, similar to control cells, and endocytosed FT9110 (Figure 2A-D). Moreover, the exposure of RAW264 cells to CNHs dispersed in FBS and PS resulted in intracellular uptake and localization of $\mathrm{CNHs}$ to the lysosomal membrane (Figure 3A-F).

\section{Assessment of FT9 I 10 uptake by flow cytometry}

The uptake of FT9110 dispersed using W-55 and H-140 increased significantly compared with the control in both dispersants. The uptake of FT9110 dispersed using W-140 increased significantly compared with that of the control in FBS. In contrast, the uptake of FT9110 dispersed using W-140 with PS was not increased compared with that of the control (Figure 4).

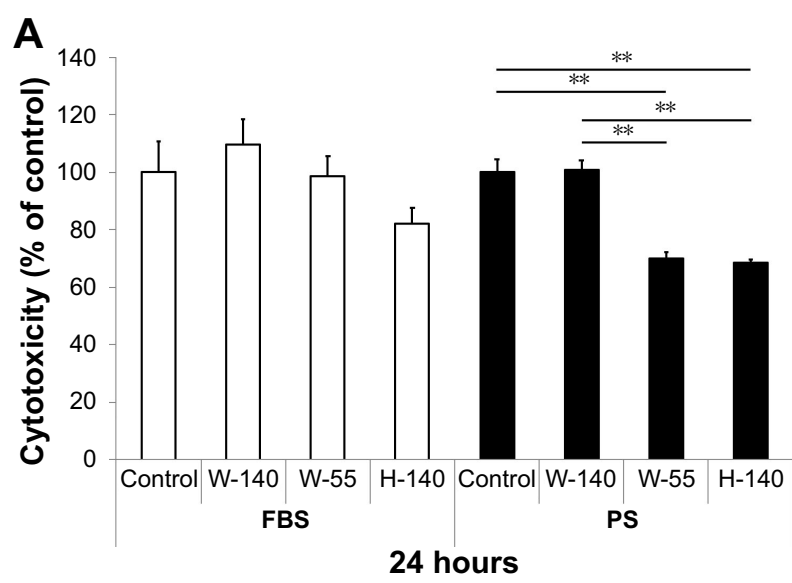

\section{Observation of cells by transmission electron microscopy (TEM)}

Cells exposed to FT9110 and CNHs were observed using TEM (Figure 5). FT9110 dispersed with W-55 was present in a bag-like structure, similar to a phagosome, exhibiting a state of entanglement. In contrast, weak uptake of FT9110 was observed in cells exposed to FT9110 dispersed with W-140, although few aggregates were observed because most particles were well dispersed. CNHs were also incorporated into the lysosomes in large amounts.

\section{Assessment of inflammatory cytokines secreted from cells after FT9IIO exposure}

A total of seven cytokines/chemokines were evaluated. Among these, interleukin (IL)-1 $\beta$, IL-10, and IL-6 were not detected by flow cytometry. In contrast, tumor necrosis factor (TNF), regulated upon activation normal $\mathrm{T}$ cell expressed and secreted (RANTES), macrophage inflammatory protein (MIP)-1 $\alpha$, and monocyte chemotactic protein (MCP)-1 showed differential secretion among groups (Figure 6). Secretion of TNF, RANTES, and MCP-1 was significantly increased when cells were exposed to FT9110 dispersed with W-55 or H-140 using FBS as a dispersant compared with that of the control, whereas MIP- $1 \alpha$ secretion decreased. In addition, the secretion of TNF and RANTES was significantly increased from cells exposed to FT9110 subjected to sonication with $\mathrm{H}-140$ and W-55 using PS as a dispersant, the secretion of MCP-1 was increased only from cells dispersed with H-140, and the secretion of MIP- $1 \alpha$ was significantly increased with W-140 and H-140. Regardless of

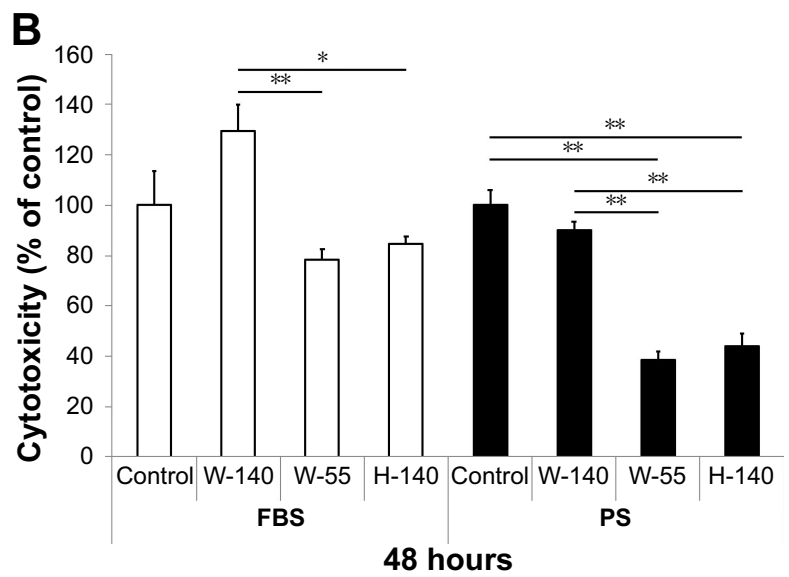

Figure I Viability of RAW264 cells exposed to FT9II0

Notes: In (A), the cell viability was measured with FT9I I0 after 24 hours. In (B), the cell viability was measured with FT9II0 after 48 hours. (A, B): FT9II0 was dispersed in FBS or PS at $100 \mu \mathrm{g} / \mathrm{mL}$. The control was medium containing each dispersant only. Data are expressed as mean $\pm S E(n=6)$. $* P<0.05 ; * * P<0.01$.

Abbreviations: FT9II0, Flotube 9II0; PS, polysorbate 80; SE, standard error. 

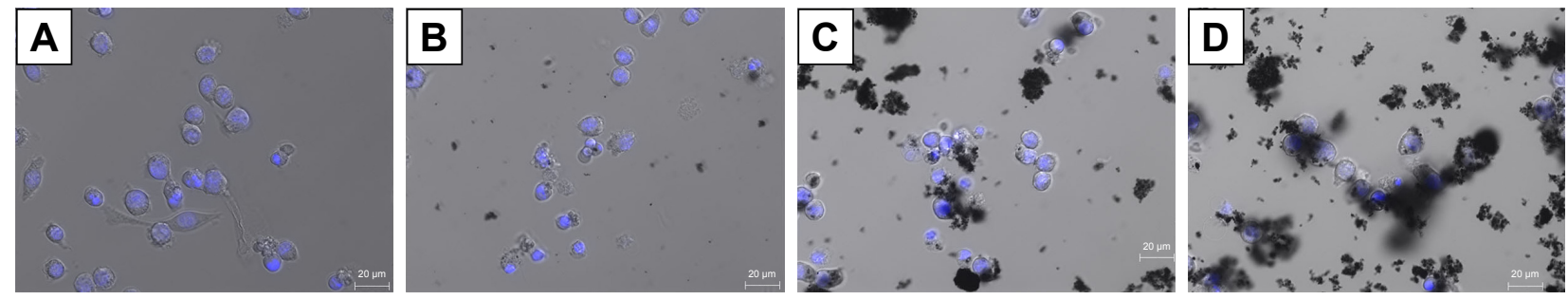

Figure 2 Live cells imaged after incubation with bisbenzimide $\mathrm{H} 33342$ fluorochrome trihydrochloride for nuclear staining.

Notes: (A) Control; (B) W-I40; (C) W-55; (D) H-I40. (B-D) RAW264 cells exposed to FT9II0 at $100 \mu g / m L$ in $0.1 \%$ PS-DPBS. The control was medium containing each dispersant only.

Abbreviations: DPBS, Dulbecco's PBS; FT91 10, Flotube 9110; PS, polysorbate 80

the dispersant, FT9110 dispersed using W-140 did not tend to yield different results from the control, although MIP- $1 \alpha$ secretion was significantly decreased compared with that of the control in FBS.

\section{Discussion}

In this study, we evaluated the safety and effects of FT9110 in macrophages. Our results provided insights into the potential applications of FT9110 by demonstrating their effects on immune system cells and cytokine/chemokine secretion.

In this study, we found that FT9110 dispersed in PS was cytotoxic after 24 hours, and our findings also suggested that both the aggregation state of the particles and the different dispersants used may have influenced cytotoxicity. There are reports that MWCNT cytotoxicity is affected by impurities. ${ }^{22}$ The current study employed FT9110 with very few impurities
(99.8\% purity) and an iron content of $<100 \mathrm{ppm}$. Although an effect of iron content in MWCNTs on cytotoxicity has been reported, ${ }^{23}$ the iron content of MWCNTs with no effect on cytotoxicity and that of FT9110 was nearly equivalent. Moreover, previous studies have shown that the uptake and cytotoxicity of MWCNTs prepared using various dispersants differed in BEAS-2B human bronchial epithelial cells and MESO-1 human malignant pleural mesothelioma cells and that the exposure of A549 lung cancer cells to CNTs dispersed with surfactant or biologically derived protein resulted in different levels of cytotoxicity and inflammatory reactions. ${ }^{24,25}$ Consistent with the abovementioned information, our findings showed that cytotoxicity was affected not by impurities contained in FT9110, but rather by the different surface modifications of FT9110 MWCNTs induced by the dispersants.
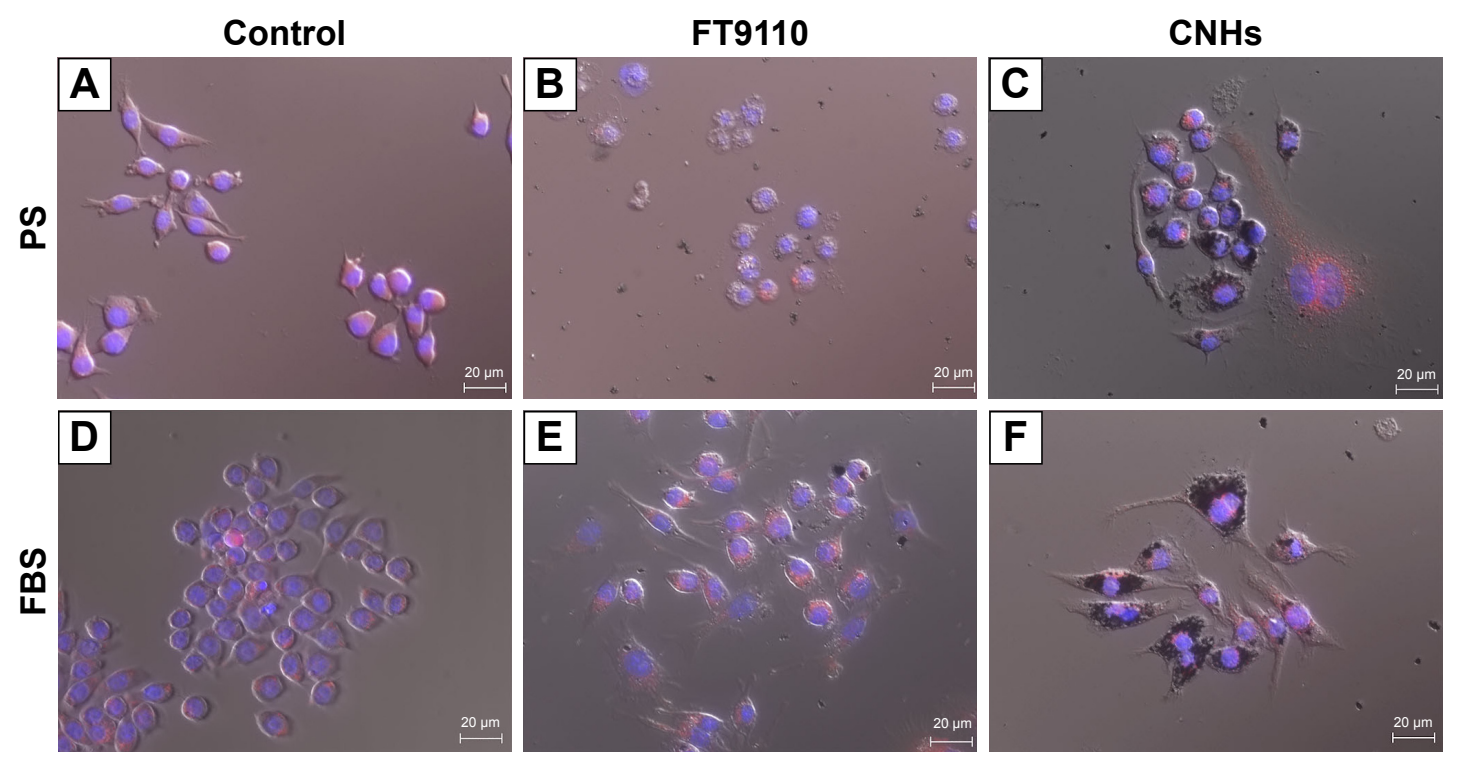

Figure 3 Live cells imaged after incubation with bisbenzimide H33342 fluorochrome trihydrochloride for nuclear staining and Cyto Painter Lysosomal Staining ab for lysosomes in dispersant (0.1\% PS-DPBS and 2\% FBS-DPBS).

Notes: (A, D) Control. RAW264 cells exposed to (B, E) FT9I 10 at $100 \mu \mathrm{g} / \mathrm{ml}$ and (C, F) CNHs at $100 \mu \mathrm{g} / \mathrm{ml}$. (A-C) RAW264 cells in $0.1 \%$ PS-DPBS and (D-F) RAW264 cells in $2 \%$ FBS-DPBS. The control was medium containing each dispersant only.

Abbreviations: CNH, carbon nanohorn; DPBS, Dulbecco's PBS; FT9I I0, Flotube 9I I0; PS, polysorbate 80. 


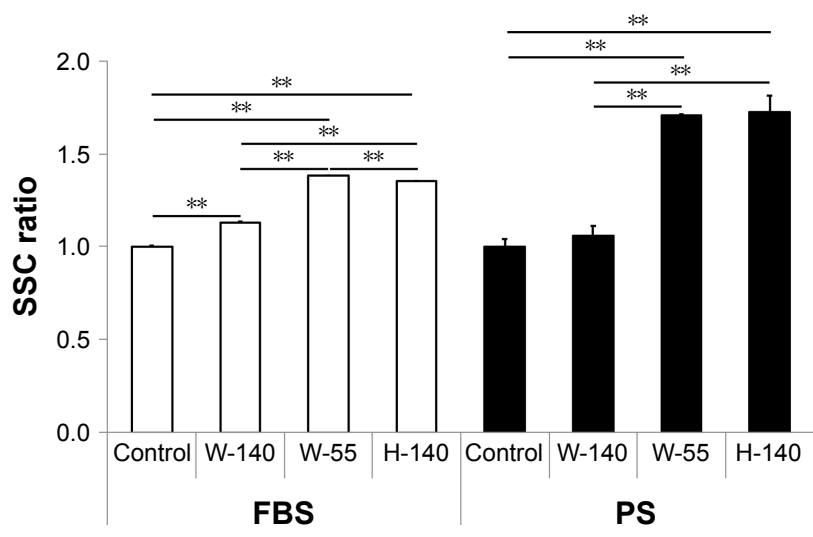

Figure 4 Uptake of FT9II0 by RAW264 cells, as demonstrated by flow cytometry.

Notes: Cultured RAW264 cells were exposed to FT9I 10 at $10 \mu \mathrm{g} / \mathrm{mL}$. The control was medium containing each dispersant only. Data are expressed as mean $\pm \mathrm{SE}$ $(\mathrm{n}=3)$. ** $\mathrm{p}<0.01$.

Abbreviations: FT9I 10, Flotube 91 I0; PS, polysorbate 80; SE, standard error; SSC, side scatter.

Notably, aggregated CNT and CNHs showed increased uptake in RAW264 cells. Previous studies have shown that various nano-sized materials are taken up into cells by endocytosis. ${ }^{26}$ In addition, RAW264 cells exposed to MWCNTs produced inflammatory cytokines and showed cytotoxicity, ${ }^{27,28}$ and when BEAS-2B human bronchial epithelial cells and small airway respiratory epithelial cells (SAEC) were exposed to MWCNTs, damage to the mitotic spindle formation was observed in a concentration-dependent manner, resulting in impaired cell division. ${ }^{29}$ In this study, aggregated CNT was taken up in RAW264 cells in large amounts, possibly inhibiting cell proliferation by producing inflammatory cytokines and blocking cell division. In contrast, the uptake of FT9110 highly dispersed in FBS was observed in RAW264 cells, although the amount was less than that of aggregated CNT or CNHs. Decreased cell viability was not observed because FT9110 did not accumulate in the cells excessively.

Interestingly, we found that CNHs dispersed in FBS and PS showed good uptake into the cells. Similarly, in a study of carbon black (CB), which is prepared using the nanocarbon material and is spherical and nano-sized, good uptake was observed in the cytoplasm through endocytosis in RAW264 cells. ${ }^{30}$ In this experiment, spherical CNHs were also potentially taken up into cells by endocytosis, similar to $\mathrm{CB}$, regardless of the dispersant. Moreover, TEM observations showed that highly dispersed FT9110 was scattered in the cytoplasm, whereas aggregated CNT was captured as aggregates in the phagosome inside the cells. We also found that CNHs were taken up into phagosomes. In our previous studies, we reported that different types of CNTs were


Figure 5 Transmission electron micrographs of RAW264 cells exposed to $100 \mu \mathrm{g} / \mathrm{mL}$ FT9I 10 and CNH uptake in dispersant (0.1\% PS-DPBS).

Notes: (A, B) Control, RAW264 cells exposed (C, D) FT9II0 sonicated by W-I40, (E, F) FT9II0 sonicated by W-55. (G, H) CNHs sonicated by W-I40. (A, C, E, G) Low magnification images. Scale bars correspond to I $\mu \mathrm{m}$. (B, D, F, H) High magnification images. Scale bars correspond to $500 \mathrm{~nm}$. The control was medium containing dispersant only. Yellow arrows indicate (C-F) FT9II 0 and (G, H) CNHs. Blue Ns indicate nuclei.

Abbreviations: CNH, carbon nanohorn; DPBS, Dulbecco's PBS; FT9II0, Flotube 9110; PS, polysorbate 80.

incorporated into cells $\mathrm{s}^{31}$ and that the same CNTs affected the uptake into cells due to the dispersed state of particles. ${ }^{21}$ In this study, like our previous study, we found that the aggregated CNT was incorporated into RAW264 cells, but that the amount of material taken up differed dramatically between highly dispersed FT9110 and CNHs showing the 
A

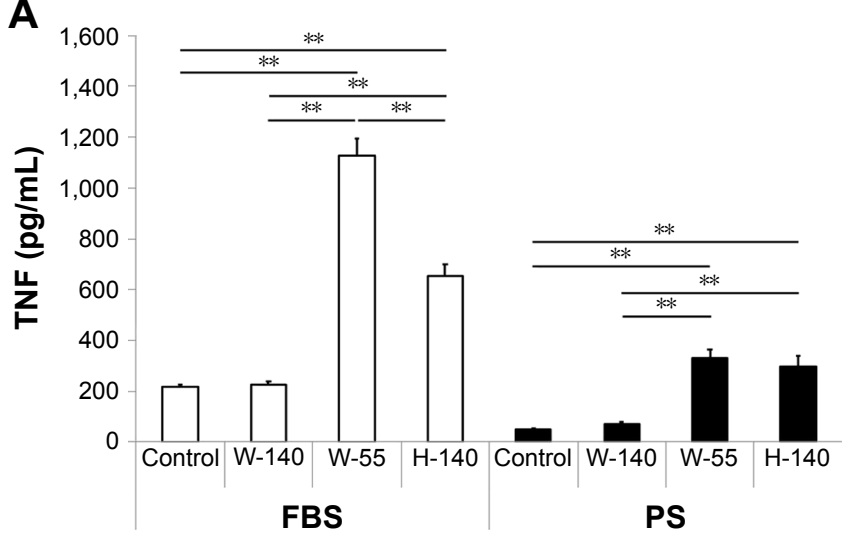

C

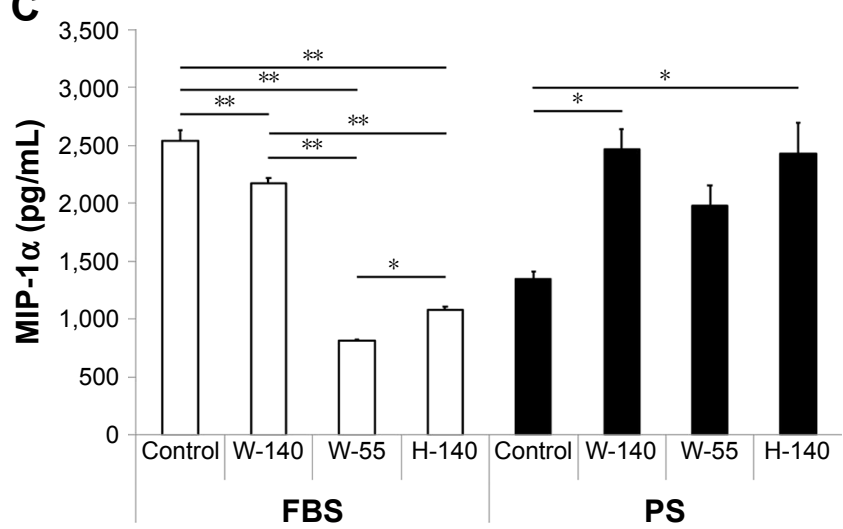

B

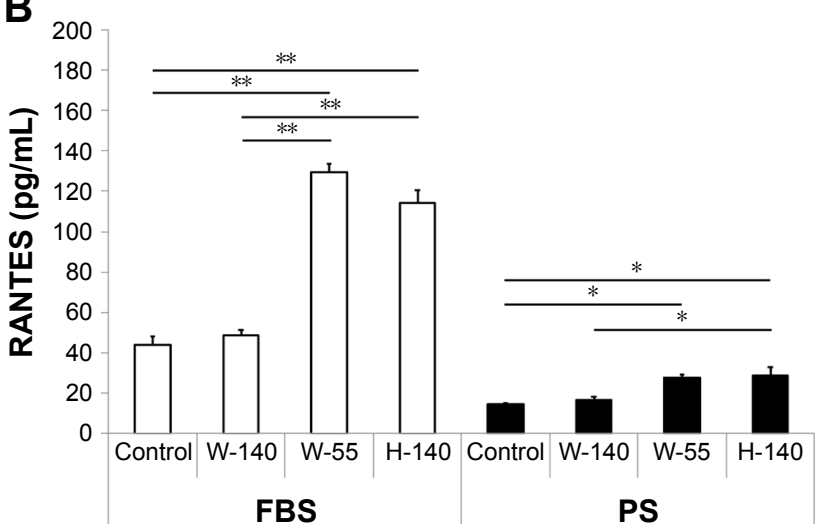

D

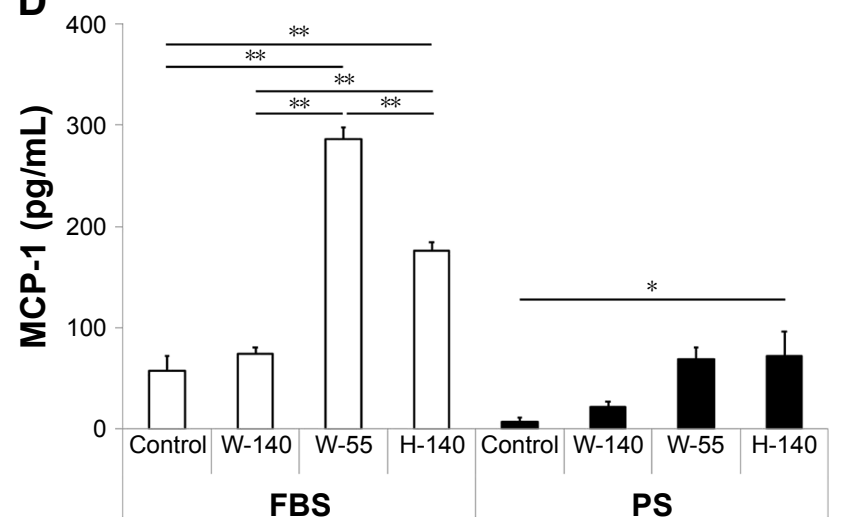

Figure 6 Measurement of inflammatory cytokine secretion by RAW264 cells after FT9I 10 exposure.

Notes: Cultured RAW264 cells were exposed to FT9I 10 at $10 \mu \mathrm{g} / \mathrm{mL}$. After 24 hours, levels of cytokine and chemokine secretion were analyzed. (A) TNF. (B) RANTES. (C) MIP-I $\alpha$. (D) MCP-I. The control was medium containing each dispersant only. Data are expressed as mean $\pm S E(n=3)$. $* P<0.05 ; * * P<0.01$.

Abbreviations: FT9II0, Flotube 91 I0; MCP, monocyte chemotactic protein; MIP, macrophage inflammatory protein; PS, polysorbate 80; RANTES, regulated upon activation normal T cell expressed and secreted; SE, standard error; TNF, tumor necrosis factor.

same degree of dispersion. Thus, these findings suggested that the mechanisms recognized by the cells differed depending on the state of aggregation and particle shape.

In our experiments, different cytotoxicity and immune responses were obtained depending on the dispersion state of FT9110 and the dispersant. Reports have described that there were differences in protein corona composition and amount among raw single-walled CNTs (SWCNTs), raw MWCNTs, and modified MWCNTs. ${ }^{32}$ We suspect that the protein corona formed on CNTs depends on the dispersing agent and moreover that the amount and kind of proteins formed by aggregation state based on the size and shape of the CNT differ greatly. The differences in cytotoxicity and immune response obtained in this study may have been due to variations in the protein corona generated by the aggregation state of FT9110 along with its surrounding dispersant and complex conditions.

Recent reports have shown that some receptors, such as the MARCO receptor, which is a type of scavenger receptor, may be involved in cellular uptake of nanomaterials, such as CNTs and CNHs, ${ }^{33-35}$ and this scavenger receptor is known to be expressed on the membrane surface of RAW264 cells. ${ }^{36}$ Scavenger receptors on RAW264 cells recognize the negative charges of CNTs, ${ }^{37}$ and MARCO receptors on J774.1 macrophages recognize proteins adsorbed on the CNT surface and induce uptake and immune responses. ${ }^{38}$ The aggregated CNT and CNHs taken up in the phagosomes in this study may be taken up via such receptors. In contrast, highly dispersed FT9110 was scattered and incorporated in the cells, but the amount of uptake was clearly less than that of aggregates. This result may be due to the fact that the surface charges and surface modifications of aggregated CNT and CNHs and highly dispersed FT9110 are different. Thus, receptors mediating uptake by macrophages that recognize carbon nanomaterials may differ, even for the same carbon nanomaterial, due to differences in particle shape, aggregation state, and surface modifications.

Cytokine secretion from RAW264 cells exposed to FT9110 varied depending on the dispersion state of FT9110 and the dispersant used. Nanoparticles such as CNTs cause 
inflammatory reactions, even when they do not affect cell proliferation, and particles of the same size may elicit different immune responses depending on the type of nanoparticles. ${ }^{39,40}$ Many receptors, such as Toll-like receptors (TLRs) and scavenger receptors, are present on macrophage cell membranes. Exposure of primary human monocyte-derived macrophages to SWCNTs demonstrated that CNTs could be recognized as pathogens, as supported by their effects on chemokine secretion induction through the TLR2/TLR4/MyD88/nuclear factor- $\kappa \mathrm{B}$ signaling pathway. ${ }^{41} \mathrm{CNTs}$ and fullerenes may be recognized as pathogens by fitting into the hydrophobic structure inside the TLR of the cell membrane in human lung carcinoma epithelial cells. ${ }^{42}$ In addition, our findings demonstrated that various cytokines could be secreted in the immune response through different receptors, such as TLRs, based on the combinations of particle size and shape, dispersant, aggregation state, and protein corona.

Cells release extracellular nucleotides and adenosine triphosphate (ATP) when exposed to inflammation or injury. ${ }^{43}$ The P2Y6 receptor, a member of the purinergic 2 receptor family, which is involved in inflammatory reactions, is expressed on the surface of RAW264 cells. Inflammatory cytokines, such as TNF $\alpha$, and chemokines, such as MIP-1 $\alpha$ and MCP-1, are produced by $\mathrm{P} 2 \mathrm{Y} 6$ receptor binding and activation with extracellular nucleotides. ${ }^{44}$ In addition, P2X4 receptors are expressed on lysosomes in RAW264 cells and may be related to phagocytosis. ${ }^{45}$ The $\mathrm{P} 2 \mathrm{X} 7$ receptor is activated by extracellular ATP and induces cell death by activating the P2X4 receptor. ${ }^{46}$ In this study, although the highly dispersed FT9110 in PS did not show cytotoxicity, secretion of MIP-1 $\alpha$ was significantly increased, and for cells treated with aggregated CNT, secretion of TNF was significantly increased when the FT9110 was dispersed in PS. Both highly dispersed FT9110 and aggregated CNT showed increased secretion of MIP- $1 \alpha$ compared with that of the control; however, the amount was lower in the FBS group than in the control. This difference may be related to the dispersion state of the particles and the surface modifications induced by the dispersing agent or dispersion method, which are involved in different mechanisms of the inflammatory reaction. Moreover, the dispersion agent or method was also involved in the inhibition of signal transduction. MIP- $1 \alpha$ is a chemokine that activates TNF, ${ }^{47}$ and TNF has been reported to be related to carcinogenesis. ${ }^{38,48}$ In this study, highly dispersed FT9110 showed reduced uptake into cells and had little effect on cell proliferation, and secretion of inflammatory cytokines, such as MIP-1 $\alpha$, was observed. In addition, highly dispersed MWCNTs localized in the cytoplasm and inhibited cell division, ${ }^{49}$ causing genomic instability and inducing DNA damage. ${ }^{50}$ Although highly dispersed FT9110 does not exhibit cytotoxicity, cellular malignancy may be caused by abnormal cell division, DNA damage, excessive secretion of cytokines associated with carcinogenesis, and other related processes.

In summary, we found that FT9110 size, shape, and dispersion state, as well as the dispersant used, caused very different reactions with regard to cellular uptake, toxicity, and immune response. These differences are presumably due to the fact that the protein corona generated by the aggregation state of CNTs and dispersant may vary, and the receptors on which CNTs act differ depending on the complex conditions to ultimately affect cell responsiveness. More detailed study is required to clarify these mechanisms along with the carcinogenicity of CNTs with the aim of establishing CNTs as therapeutic biomaterials. Moreover, to apply CNTs as biomaterials, it will be necessary to evaluate the shapes and dispersion states of different particles, the dispersant used, and the optimal surface modifications for different purposes.

\section{Conclusion}

Highly dispersed and aggregated CNT showed different degrees of uptake into cells and localization in the cells, and the inflammatory cytokines expressed following the exposure to FT9110 varied greatly depending on the aggregation state of the particles and the dispersing agent. Evaluation of the incorporation of FT9110 and CNHs into the cells showed that highly dispersed FT9110 and aggregated CNT or CNHs were taken up intracellularly, although the mechanisms of incorporation varied. Thus, receptors related to intracellular uptake of individual particles and immune responses may differ. This study was performed in vitro; therefore, it is necessary to conduct animal experiments to evaluate CNT uptake and immune responses while considering complicated biological reactions. Because the cellular response varies depending on the state of aggregation and the dispersant used, even for the same type of material, further studies are necessary to clarify the effects of CNT shape, dispersion state, dispersants used, and surface modification to determine the carcinogenicity of CNTs and develop CNTs for clinical applications.

\section{Acknowledgments}

We would like to thank the staff at the Department of Instrumental Analysis at the Research Center for Supports to Advanced Science of Shinshu University for their assistance. We would like to thank Editage (www.editage.jp) for English 
language editing. This study was supported by the Japan Society for the Promotion of Science (JSPS) KAKENHI (grant nos 17H01584, 25350524, 25462365, and 24241045) and Japan Agency for Medical Research and Development (AMED) (grant no JP17km1009852). Chika Kuroda and Katsuya Ueda are co-first authors for this study.

\section{Disclosure}

The authors report no conflicts of interest in this work.

\section{References}

1. de Volder MF, Tawfick SH, Baughman RH, Hart AJ. Carbon nanotubes: present and future commercial applications. Science. 2013; 339(6119):535-539.

2. Shvedova AA, Kisin ER, Porter D, et al. Mechanisms of pulmonary toxicity and medical applications of carbon nanotubes: Two faces of Janus? Pharmacol Ther. 2009;121(2):192-204.

3. Saito N, Haniu H, Usui Y, et al. Safe clinical use of carbon nanotubes as innovative biomaterials. Chem Rev. 2014;114(11):6040-6079.

4. Hartman KB, Hamlin DK, Wilbur DS, Wilson LJ.211AtCl@US-tube nanocapsules: a new concept in radiotherapeutic-agent design. Small. 2007;3(9):1496-1499.

5. Ruggiero A, Villa $\mathrm{CH}$, Bander E, et al. Paradoxical glomerular filtration of carbon nanotubes. Proc Natl Acad Sci U S A. 2010;107(27): 12369-12374.

6. Johnston HJ, Hutchison GR, Christensen FM, et al. A critical review of the biological mechanisms underlying the in vivo and in vitro toxicity of carbon nanotubes: The contribution of physico-chemical characteristics. Nanotoxicology. 2010;4(2):207-246.

7. Sato Y, Yokoyama A, Nodasaka Y, et al. Long-term biopersistence of tangled oxidized carbon nanotubes inside and outside macrophages in rat subcutaneous tissue. Sci Rep. 2013;3:2516.

8. Hussain S, Sangtian S, Anderson SM, et al. Inflammasome activation in airway epithelial cells after multi-walled carbon nanotube exposure mediates a profibrotic response in lung fibroblasts. Part Fibre Toxicol. 2014;11:28.

9. Chen T, Nie H, Gao X, et al. Epithelial-mesenchymal transition involved in pulmonary fibrosis induced by multi-walled carbon nanotubes via TGF-beta/Smad signaling pathway. Toxicol Lett. 2014;226(2): $150-162$.

10. Broz P, Dixit VM. Inflammasomes: mechanism of assembly, regulation and signalling. Nat Rev Immunol. 2016;16(7):407-420.

11. Donaldson K, Murphy FA, Duffin R, Poland CA. Asbestos, carbon nanotubes and the pleural mesothelium: a review of the hypothesis regarding the role of long fibre retention in the parietal pleura, inflammation and mesothelioma. Part Fibre Toxicol. 2010;7:5.

12. Bellucci S, Chiaretti M, Cucina A, Carru GA, Chiaretti AI. Multiwalled carbon nanotube buckypaper: toxicology and biological effects in vitro and in vivo. Nanomedicine. 2009;4(5):531-540.

13. Luo M, Chen P, Wang JJ, et al. The cytotoxicity of oxidized multiwalled carbon nanotubes on macrophages. Science China Chemistry. 2016;59(7):918-926.

14. Sato Y, Yokoyama A, Shibata K, et al. Influence of length on cytotoxicity of multi-walled carbon nanotubes against human acute monocytic leukemia cell line THP-1 in vitro and subcutaneous tissue of rats in vivo. Mol Biosyst. 2005;1(2):176-182.

15. Gottardi R, Douradinha B. Carbon nanotubes as a novel tool for vaccination against infectious diseases and cancer. J Nanobiotechnology. 2013;11:30.

16. Poland CA, Duffin R, Kinloch I, et al. Carbon nanotubes introduced into the abdominal cavity of mice show asbestos-like pathogenicity in a pilot study. Nat Nanotechnol. 2008;3(7):423-428.
17. Takagi A, Hirose A, Nishimura T, et al. Induction of mesothelioma in p53 mouse by intraperitoneal application of multi-wall carbon nanotube. J Toxicol Sci. 2008;33(1):105-116.

18. Haniu H, Saito N, Matsuda Y, et al. Elucidation mechanism of different biological responses to multi-walled carbon nanotubes using four cell lines. Int J Nanomedicine. 2011;6:3487-3497.

19. Nagai H, Okazaki Y, Chew SH, et al. Intraperitoneal administration of tangled multiwalled carbon nanotubes of $15 \mathrm{~nm}$ in diameter does not induce mesothelial carcinogenesis in rats. Pathol Int. 2013; 63(9):457-462.

20. Ruan B, Jacobi AM. Ultrasonication effects on thermal and rheological properties of carbon nanotube suspensions. Nanoscale Res Lett. 2012;7:127.

21. Kuroda C, Haniu H, Ajima K, et al. The Dispersion State of Tangled Multi-Walled Carbon Nanotubes Affects Their Cytotoxicity. Nanomaterials. 2016;6(11):E219:219.

22. Cui HF, Vashist SK, Al-Rubeaan K, Luong JH, Sheu FS. Interfacing carbon nanotubes with living mammalian cells and cytotoxicity issues. Chem Res Toxicol. 2010;23(7):1131-1147.

23. Haniu H, Matsuda Y, Takeuchi K, Kim YA, Hayashi T, Endo M. Proteomics-based safety evaluation of multi-walled carbon nanotubes. Toxicol Appl Pharmacol. 2010;242(3):256-262.

24. Haniu H, Saito N, Matsuda Y, et al. Effect of dispersants of multiwalled carbon nanotubes on cellular uptake and biological responses. Int J Nanomedicine. 2011;6:3295-3307.

25. Horie M, Stowe M, Tabei M, et al. Dispersant affects the cellular influences of single-wall carbon nanotube: the role of CNT as carrier of dispersants. Toxicol Mech Methods. 2013;23(5):315-322.

26. Conner SD, Schmid SL. Regulated portals of entry into the cell. Nature. 2003;422(6927):37-44.

27. van Berlo D, Wilhelmi V, Boots AW, et al. Apoptotic, inflammatory, and fibrogenic effects of two different types of multi-walled carbon nanotubes in mouse lung. Arch Toxicol. 2014;88(9):1725-1737.

28. Oberdörster G, Oberdörster E, Oberdörster J. Nanotoxicology: an emerging discipline evolving from studies of ultrafine particles. Environ Health Perspect. 2005;113(7):823-839.

29. Siegrist KJ, Reynolds SH, Kashon ML, et al. Genotoxicity of multiwalled carbon nanotubes at occupationally relevant doses. Part Fibre Toxicol. 2014;11:6.

30. Kong H, Zhang Y, Li Y, et al. Size-dependent cytotoxicity of nanocarbon blacks. Int J Mol Sci. 2013;14(11):22529-22543.

31. Haniu H, Saito N, Matsuda Y, et al. Biological responses according to the shape and size of carbon nanotubes in BEAS-2B and MESO-1 cells. Int J Nanomedicine. 2014;9:1979-1990.

32. Shannahan JH, Brown JM, Chen R, et al. Comparison of nanotubeprotein corona composition in cell culture media. Small. 2013;9(12): 2171-2181.

33. Kanno S, Furuyama A, Hirano S. A murine scavenger receptor MARCO recognizes polystyrene nanoparticles. Toxicol Sci. 2007;97(2): 398-406.

34. Hirano S, Fujitani Y, Furuyama A, Kanno S. Macrophage receptor with collagenous structure (MARCO) is a dynamic adhesive molecule that enhances uptake of carbon nanotubes by CHO-K1 cells. Toxicol Appl Pharmacol. 2012;259(1):96-103.

35. Nakayama M. Macrophage Recognition of Crystals and Nanoparticles. Front Immunol. 2018;9:103.

36. Fitzgerald ML, Moore KJ, Freeman MW, Reed GL. Lipopolysaccharide induces scavenger receptor A expression in mouse macrophages: a divergent response relative to human THP-1 monocyte/macrophages. J Immunol. 2000;164(5):2692-2700.

37. Wang X, Guo J, Chen T, et al. Multi-walled carbon nanotubes induce apoptosis via mitochondrial pathway and scavenger receptor. Toxicol In Vitro. 2012;26(6):799-806.

38. Hirano S, Kanno S, Furuyama A. Multi-walled carbon nanotubes injure the plasma membrane of macrophages. Toxicol Appl Pharmacol. 2008;232(2):244-251. 
39. Dumortier H. When carbon nanotubes encounter the immune system: desirable and undesirable effects. Adv Drug Deliv Rev. 2013;65(15): 2120-2126.

40. Liu D, Yang F, Xiong F, Gu N. The Smart Drug Delivery System and Its Clinical Potential. Theranostics. 2016;6(9):1306-1323.

41. Mukherjee SP, Bondarenko O, Kohonen P, et al. Macrophage sensing of single-walled carbon nanotubes via Toll-like receptors. Sci Rep. 2018;8(1):1115

42. Turabekova M, Rasulev B, Theodore M, Jackman J, Leszczynska D, Leszczynski J. Immunotoxicity of nanoparticles: a computational study suggests that CNTs and C60 fullerenes might be recognized as pathogens by Toll-like receptors. Nanoscale. 2014;6(7):3488-3495.

43. Boeynaems JM, Communi D, Gonzalez NS, Robaye B. Overview of the P2 receptors. Semin Thromb Hemost. 2005;31(2):139-149.

44. Zhang Z, Wang Z, Ren H, et al. P2Y(6) agonist uridine 5'-diphosphate promotes host defense against bacterial infection via monocyte chemoattractant protein-1-mediated monocytes/macrophages recruitment. J Immunol. 2011;186(9):5376-5387.
45. Qureshi OS, Paramasivam A, Yu JC, Murrell-Lagnado RD. Regulation of $\mathrm{P} 2 \times 4$ receptors by lysosomal targeting, glycan protection and exocytosis. J Cell Sci. 2007;120(Pt 21):3838-3849.

46. Kawano A, Tsukimoto $\mathrm{M}$, Noguchi $\mathrm{T}$, et al. Involvement of $\mathrm{P} 2 \times 4$ receptor in $\mathrm{P} 2 \times 7$ receptor-dependent cell death of mouse macrophages. Biochem Biophys Res Commun. 2012;419(2):374-380.

47. Dorner BG, Scheffold A, Rolph MS, et al. MIP-1alpha, MIP-1beta, RANTES, and ATAC/lymphotactin function together with IFN-gamma as type 1 cytokines. Proc Natl Acad Sci U S A. 2002;99(9):6181-6186.

48. Wu Y, Zhou BP. TNF-alpha/NF-kappaB/Snail pathway in cancer cell migration and invasion. Br J Cancer. 2010;102(4):639-644.

49. Asakura M, Sasaki T, Sugiyama T, et al. Genotoxicity and cytotoxicity of multi-wall carbon nanotubes in cultured Chinese hamster lung cells in comparison with chrysotile A fibers. J Occup Health. 2010;52(3): 155-166.

50. Pacurari M, Yin XJ, Ding M, et al. Oxidative and molecular interactions of multi-wall carbon nanotubes (MWCNT) in normal and malignant human mesothelial cells. Nanotoxicology. 2008;2(3):155-170.
International Journal of Nanomedicine

\section{Publish your work in this journal}

The International Journal of Nanomedicine is an international, peerreviewed journal focusing on the application of nanotechnology in diagnostics, therapeutics, and drug delivery systems throughout the biomedical field. This journal is indexed on PubMed Central, MedLine, CAS, SciSearch $\AA$, Current Contents ${ }^{\circledR} /$ Clinical Medicine,

\section{Dovepress}

Journal Citation Reports/Science Edition, EMBase, Scopus and the Elsevier Bibliographic databases. The manuscript management system is completely online and includes a very quick and fair peer-review system, which is all easy to use. Visit http://www.dovepress.com/ testimonials.php to read real quotes from published authors. 